\title{
Dynamics of ocular surface topography
}

\begin{abstract}
Purpose To investigate fluctuations in the ocular surface, we used high-speed videokeratoscopy $(50 \mathrm{~Hz})$ to measure the dynamics of the ocular surface topography. Methods Ocular surface height difference maps were computed to illustrate the changes in the tear film in the inter-blink interval. Topography data were used to derive the ocular surface wavefront aberrations up to the fourth radial order of the Zernike polynomial expansion. We examined the ocular surface dynamics and temporal changes in the ocular surface wavefront aberrations in the inter-blink interval.

Results During the first $0.5 \mathrm{~s}$ following a blink, the ocular surface height at the upper edge of the topography map increased by about $2 \mu \mathrm{m}$. Temporal changes occurred for some ocular surface wavefront aberrations and appeared to be related to changes in the distribution of tear film.

Conclusion In the clinical measurement of ocular surface topography using videokeratoscopy or optics of the eye using wavefront sensors, care should be taken to avoid the initial tear film build-up phase following a blink to achieve more consistent results.
\end{abstract}

Eye (2007) 21, 624-632. doi:10.1038/sj.eye.6702293; published online 21 April 2006

Keywords: ocular surface topography; ocular surface aberrations; ocular microfluctuations; high-speed videokeratoscopy

\section{Introduction}

The pre-corneal tear film provides a smooth, regular anterior surface of the eye. Tear film builds up after a blink and becomes unstable over time, finally breaking up if the eye is left open for a sufficient period of time. ${ }^{1-3}$ Local changes in the tear film or tear irregularity that overlap the pupil area can introduce additional aberrations into the optics of the eye; hence, instability of the tear film will cause changes in the optical quality of the eye.

Corneal topography techniques based on the Placido disk principle use the tear film as a convex mirror to view the first Purkinje image; therefore, they measure the pre-corneal tear film surface and not the corneal surface. If the tear film is stable, continuous, and has constant radial thickness, then we can assume that the measurement based on the Placido technique is closely approximating the corneal surface.

There have been numerous studies of the changes in the topography of the ocular surface in the inter-blink period using

videokeratoscopy. Nemeth et $a l^{4}$ measured the ocular surface topography with a videokeratoscope in the interval between 5 and $10 \mathrm{~s}$ after a complete blink and showed significant differences in ocular surface topography (in terms of surface regularity index) whereas the ocular surface refractive power remained unaltered. A significant increase in both total and ocular surface aberrations 10 and $20 \mathrm{~s}$ after blink has been reported for larger pupil sizes $(>3.5 \mathrm{~mm})$ by Montes-Mico et $a l^{5}$ and they suggested that these optical changes were caused by increasing irregularity in the tear film with time.

With the emerging technology of high-speed videokeratoscopy, it is possible to acquire information on the dynamic changes of the ocular surface. High-speed videokeratoscopy was developed to automatically capture consecutive ocular surface topography and hence this technique can be used to examine the tear film dynamics. In the investigation of tear film stability, this technique was used as a noninvasive and objective method for the clinical assessment of tear film stability at a frequency of $1 \mathrm{~Hz}^{6-8}$ In other studies, the changes of higher order ocular surface wavefront aberrations were investigated in normal and dry eyes., ${ }^{3,9}$ The higher order aberrations were found to first decrease immediately after a blink, reaching a minimum $6 \mathrm{~s}$ post-blink, and then to increase steadily. ${ }^{3}$ 
Nemeth et $a l^{2}$ used high-speed videokeratoscopy to assess tear film regularity changes at a sampling frequency of $4 \mathrm{~Hz}$. In their study, the tear film build-up time was measured based on the changes in the surface regularity and asymmetry indices and it was found that tear film was most stable 3-10 s after a blink. However, the limitations of the surface regularity and asymmetry indices as an assessment of tear film stability by highspeed videokeratoscopy were recently reported by Iskander et $a^{10}$ They used a sampling rate of $50 \mathrm{~Hz}$ in the acquisition of videokeratographs and proposed a technique for estimating the tear film build-up time based on the RMS of the error of the parametric model fit to the ocular surface.

In this study, the dynamics of ocular surface topography were measured using a high-speed videokeratoscope at a sampling frequency of $50 \mathrm{~Hz}$. Relative changes in the tear film during the inter-blink interval were visualised by computing the ocular surface height difference maps over time. This study aimed to investigate the temporal changes in the tear film topography and the variations in the ocular surface wavefront aberrations in the period between blinks.

\section{Methods}

\section{Data acquisition}

Ocular surface topography was obtained by using a highspeed videokeratoscope system based on the Medmont E300 videokeratoscope (Medmont Pty Ltd, Camberwell, VIC, Australia). The main components of this system have been described by Iskander et al. ${ }^{11}$

A group of 10 young subjects participated in this study with mean ages of 24.8 years (range between 21 and 30 years). Informed consent was obtained from all participates and the experiment conformed to a protocol approved by the Human Research Ethics Committee of the university. Seven subjects were emmetropes and three were myopes (less than $-5 \mathrm{D}$ ). All subjects had normal, healthy eyes, and none had dry eye symptoms.

The ocular surface topography of the left eye was measured at a sampling frequency of $50 \mathrm{~Hz}$ and for each acquisition we collected a 40 s continuous record (ie 2000 images). The subjects did not wear contact lenses or spectacles and were instructed to focus on the instrument's internal fixation target. The right eye (untested) had a free view past the instrument. Subjects were instructed to have natural blinks whenever necessary and to breathe at $0.5 \mathrm{~Hz}$, guided by an electronic metronome. A breathing rate of $0.5 \mathrm{~Hz}$ was chosen for ease of identification of this frequency component in subject power spectrum analysis. When two similar frequencies arise in power spectra, discrimination of two separate peaks can be difficult. Subjects were also instructed not to deliberately open their eyes wide, but to look naturally at the fixation target. By instructing subjects to blink naturally and not deliberately widen their eyelid aperture, we assessed ocular surface dynamics in a relatively natural state.

The pulse signal of the subject was simultaneously collected by a MacLab/4s and Bio Amp (ADInstruments Pty Ltd, Castal Hill, NSW, Australia), which is designed to record the electrocardiogram (ECG) using electrodes connected to both wrists and the right ankle of the subject. The pulse signal was measured at a sampling frequency of $40 \mathrm{~Hz}$ for a duration of $40 \mathrm{~s}$. The frequency range of primary interest is normally less than $2 \mathrm{~Hz}$, so the sampling rates of both instruments were well above the Nyquist frequency. The acquired pulse signal was used to derive the instantaneous heart rate. ${ }^{12}$

Instantaneous heart rate is the cyclic fluctuation in the time delay between each heart beat (heart rate increases slightly during inspiration and reduces during expiration), whereas pulse rate is simply the number of heart beats per minute. Three simultaneous recordings of pulse signals and ocular surface topography data were collected for each of the subjects.

\section{Data analysis}

\section{Surface characteristics}

The high-speed videokeratoscopy data were used to calculate the ocular surface height maps that depict the relative height of the tear film from the apex of the ocular surface. Ocular surface height maps were centred on the videokeratoscope axis (vertex normal) and will have zero value at the centre. Ocular surface height difference maps were derived by subtracting a baseline ocular surface height map from a series of sequential maps to reveal and highlight the changes in the distribution of tear film. The height difference map provides 2D information and reflects the relative changes in the thickness of tear film that covers the ocular surface provided that the underlying corneal topography is stable and does not change during the data acquisition. To study the tear dynamics in the inter-blink interval, we normally chose as the baseline map the first available videokeratograph directly after a blink that had complete information and a good quality ring pattern (defined at $t=0$ ). All the subsequent ocular surface images leading up to the next blink were subtracted from this baseline map (ie surface difference maps) to investigate the tear dynamics such as tear flow and tear distribution.

Ocular surface height difference maps will have zero values at the centre of the map, irrespective of the actual thickness of the underlying tear film. Therefore, tear thickness changes at this location cannot be measured 
and height difference maps only reflect the changes in the ocular surface height relative to the reference map (ie acquired immediately after the blink).

In this study, our subjects were instructed to focus on the target naturally without widening their aperture purposely. Therefore, the location of the eyelids was normally $3-4 \mathrm{~mm}$ from the centre of the pupil. The surface height maps were generally $6-8 \mathrm{~mm}$ wide vertically and $9-11 \mathrm{~mm}$ wide horizontally.

\section{Ocular surface aberrations}

Each videokeratograph was also used to estimate the ocular surface wavefront aberrations for a $5 \mathrm{~mm}$ entrance pupil diameter. This has been achieved by performing a 3 D ray trace at the line of sight that was assumed to be at the centre of the entrance pupil. The latter was estimated from the videokeratoscopy raw digital image. Compared with the height difference maps, the Zernike wavefront aberrations analysis has the advantage of providing a mathematical analysis of shape changes in the surface of the tear film. The effect of microfluctuations is also minimised as it is normally assumed that the pupil does not change its position with respect to geometrical centre of cornea during the measurement. The wavefront aberrations were decomposed into a set of Zernike polynomials up to the fourth radial order. The timevarying ocular surface wavefront aberrations, $w(\rho, \theta, t)$, are mathematically modelled, ${ }^{13}$

$(\rho, \theta ; t)=\sum_{i=1}^{15} a_{i}(t) Z_{i}(\rho, \theta)+\varepsilon(\rho, \theta ; t)$

where $a_{i}(t), i=1,2, \ldots, 15$, are the time-varying Zernike coefficient signals and $Z_{i}(\rho, \theta)$ are the orthogonal Zernike polynomials. Note that in the above equation, the wavefront is a function of the radial distance $\rho$ and angle $\theta$ as well as the time $t$. The instrument and modelling noise is denoted by $\varepsilon(\rho, \theta, t)$.

The blink artefacts from the ocular surface wavefront aberration coefficient signals were removed using a third-order polynomial interpolation technique. Specifically, an algorithm was applied to the ocular surface wavefront signals to find the locations of the blinks where the ocular surface wavefront aberration coefficients are zero (the centre of the pupil is undetectable). Problems were encountered when using some images captured during the downward and upward phases of a blink even though the pupil centres of these images were detectable. The topography information could be incomplete because of the low position of the eyelid, and in some cases the images were blurred or distorted owing to the lid and eye movement. Often we found that 2-3 images (0.04-0.06 s) immediately before and after each blink either had incomplete topography information or blurred ring pattern, which could lead to inaccurate estimations of the ocular surface wavefront aberration coefficients and abrupt changes in signal amplitude. Therefore, we decided to ignore three images immediately before and after each blink and interpolate according to the remaining signal information.

\section{Eye position}

When the ocular surface topography data were acquired, the high-speed videokeratoscope also monitored the anterior-posterior eye position changes. The distances between the ocular surface apex and the instrument position sensing system (apex distance) were recorded during the measurements and these data were later used to investigate the microfluctuations of the anteriorposterior eye position.

\section{Power spectrum analysis}

Before temporal or frequency analyses of the Zernike coefficient signal is conducted, the signals are first detrended by removing a linear trend and then bandpass filtered to extract the frequency range of interest $(0.1-2 \mathrm{~Hz})$. Detrending is a standard signal processing procedure that removes the DC component from a signal. It is performed because the fluctuations in each of the Zernike aberration signals are of interest rather than their actual values. As a band-pass filter, a digital filter was used to remove the low-frequency $(<0.02 \mathrm{~Hz})$ and high-frequency $(>2 \mathrm{~Hz}$ ) components contained within the signal.

For each of the 14 Zernike coefficient signals (piston excluded) $a_{i}(t), i=2,3, \ldots, 15$, a procedure of power spectrum estimation techniques based on the fast Fourier transform (FFT) was used to estimate the power spectra of the aberration signals. The same procedure of spectral analysis was applied to the pulse and the instantaneous heart rate signals. The spectral representations of aberration and pulse signals were then used to determine the similarities (common frequency peaks) between the signals.

\section{Results}

Within the first $0.5 \mathrm{~s}$ after the blink, the ocular surface height significantly increased at the top of the topography map and decreased in the inferior region of the map. The ocular surface topography generally became relatively stable $2-3 \mathrm{~s}$ post-blink. There were no clear trends in ocular surface dynamics between subjects in the subsequent inter-blink period up to $4.5 \mathrm{~s}$ postblink. Few ocular surface high-order aberrations showed significant changes in the inter-blink interval. 


\section{Changes in tear film surface height}

Group ocular surface height changes were examined and the mean changes at the vertical meridian $3 \mathrm{~mm}$ above and below centre are presented in Figures 1 and 2. The mean changes in the ocular surface height found within the first $0.5 \mathrm{~s}$ after a blink were a $2 \mu \mathrm{m}( \pm 2 \mu \mathrm{m})$ increase (ie tear thickening) $3 \mathrm{~mm}$ above the centre (Figure 1) and less than $0.5 \mu \mathrm{m}( \pm 1 \mu \mathrm{m})$ decrease (ie tear thinning) $3 \mathrm{~mm}$ below the centre (Figure 2). The group mean surface height changes in the post-blink interval were smaller than many individual results because of averaging of significant intra- and inter-subject variability. The oneway repeated measures analysis of variance (ANOVA) showed that the group ocular surface height $3 \mathrm{~mm}$ above the centre in the blink interval of $4.5 \mathrm{~s}$ significantly increased $(\mathrm{F}=1.691, \mathrm{df}=44, P=0.006)$. However, no significant changes over time were found in the group ocular surface height $3 \mathrm{~mm}$ below the centre $(\mathrm{F}=1.025$, $\mathrm{df}=44, P=0.434)$.

To illustrate the ocular surface dynamics in the interblink interval, ocular height difference maps for subject DZ representing the difference between the first and second maps, first and 12th, and first and 22nd up to $3.82 \mathrm{~s}$ post-blink are shown in Figure 3. If we assume that the underlying corneal topography is stable, then changes in surface height should reflect relative changes in the tear film thickness relative to the centre of the map. In this example, thickening at the superior edge of the map was observed after the blink as the tears presumably

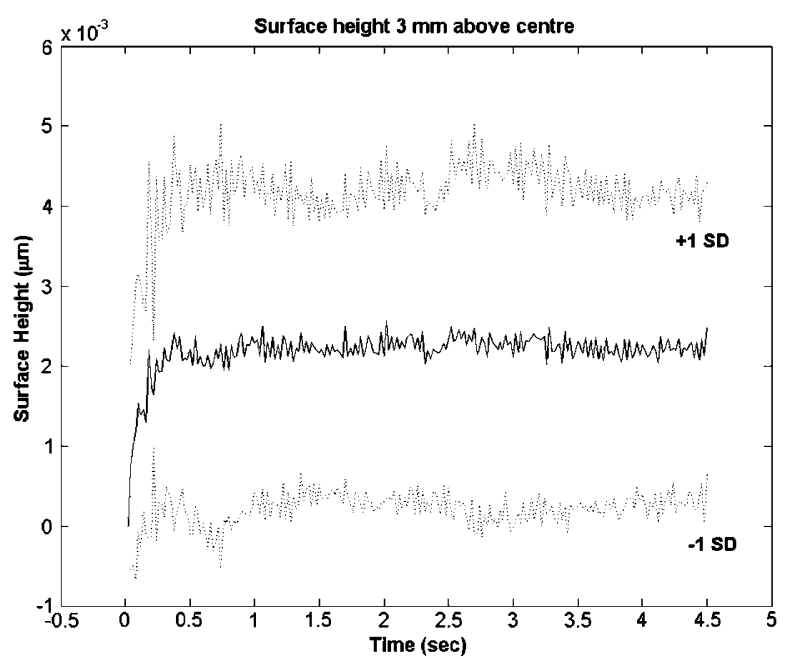

Figure 1 Group ocular surface height changes in the vertical meridian $3 \mathrm{~mm}$ above centre. Data are the mean of multiple signals for nine subjects (only the inter-blink intervals of at least $4.5 \mathrm{~s}$ were included in this analysis). Dotted lines indicate $\pm 1 \mathrm{SD}$ error bars. On the $y$ axis, positive values represent increased height (ie tear thickening) and negative values represent decreased height (ie tear thinning) relative to the surface height immediately after the blink $(t=0)$. moved upward following the upward movement of the upper eyelid. Up until $0.62 \mathrm{~s}$ following the blink, the superior region of the map became progressively thicker, with $4-6 \mu \mathrm{m}$ increase. At the same time, the inferior region of the map became thinner by $2-3 \mu \mathrm{m}$ compared with the baseline map taken immediately after the blink. In the next phase of the post-blink interval (0.62-3.82 s), the ocular surface height decreased slightly in the superior region of the map and increased slightly in the inferior region. This presumably reflects a slow downward flow of tears.

To explore the tear film dynamics during the tear film build-up phase immediately after each blink with better time resolution ( $0.04 \mathrm{~s})$, ocular surface height difference maps for subject DZ representing the difference between the first and second maps, first and fourth, and first and sixth up to $0.62 \mathrm{~s}$ post-blink are shown in Figure 4. There is a rapid increase in ocular surface height from 0.02 to $0.26 \mathrm{~s}$ post-blink in the superior region of the map. From 0.26 to $0.62 \mathrm{~s}$ post-blink, the surface height is relatively stable with a slight increase in the height continuing to occur at the superior edge of the map.

The changes in the surface height during the 'interblink' phase (Figure 3, 0.62-3.82 s post-blink) were comparatively smaller than those during the 'build-up' phase (up to $0.62 \mathrm{~s}$ post-blink). The tears appeared to flow downwards in this period because the inferior region of the map became higher and the superior region

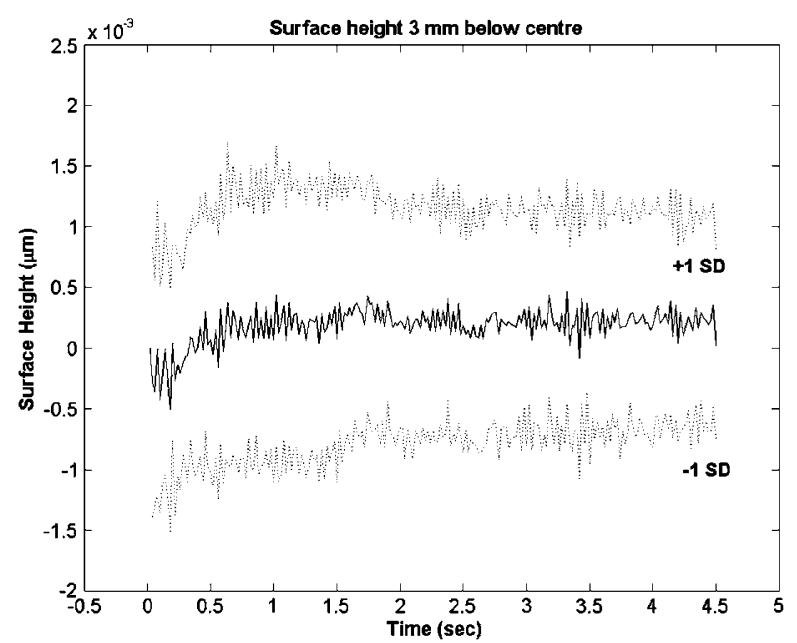

Figure 2 Group ocular surface height changes at the vertical meridian $3 \mathrm{~mm}$ below the centre. Data are the mean of multiple signals for nine subjects (only the inter-blink intervals of at least $4.5 \mathrm{~s}$ were included in this analysis). Dotted lines indicate $\pm 1 \mathrm{SD}$ error bars. On the $y$ axis, positive values represent increased height (ie tear thickening) and negative values represent decreased height (ie tear thinning) relative to the surface height immediately after the blink $(t=0)$. 


\section{Total inter-blink period}

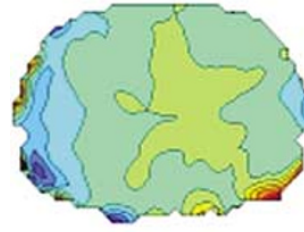

$\mathrm{t}=0.02$

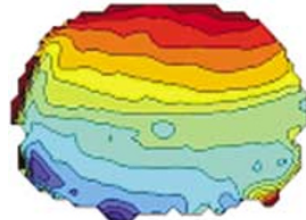

$\mathrm{t}=0.82$

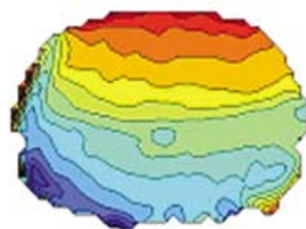

$\mathrm{t}=1.62$

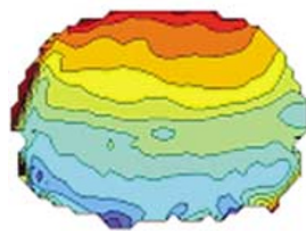

$\mathrm{t}=2.42$

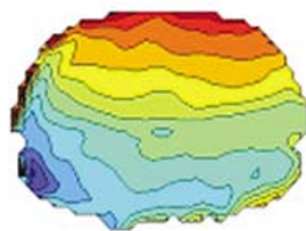

$\mathrm{t}=3.22$

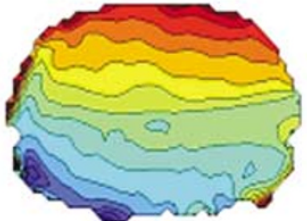

$\mathrm{t}=0.22$

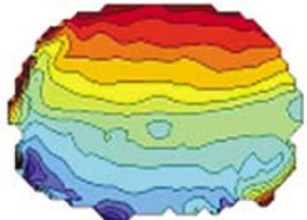

$\mathrm{t}=1.02$

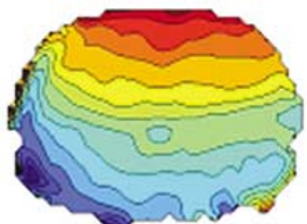

$\mathrm{t}=1.82$

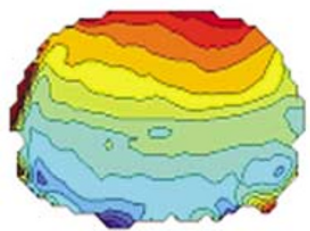

$\mathrm{t}=2.62$

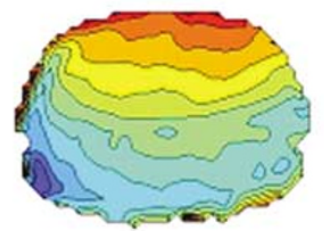

$\mathrm{t}=3.42$

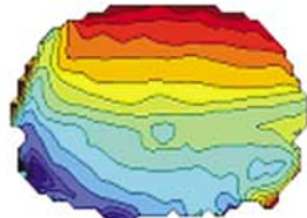

$\mathrm{t}=0.42$

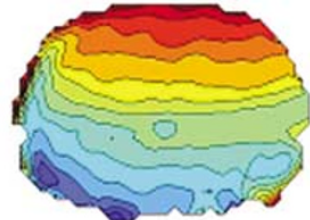

$\mathrm{t}=1.22$

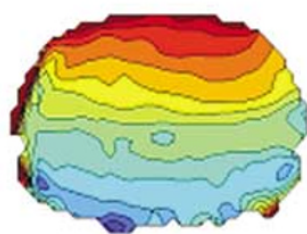

$\mathrm{t}=2.02$

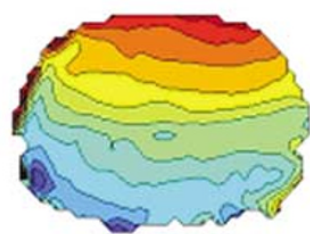

$\mathrm{t}=2.82$

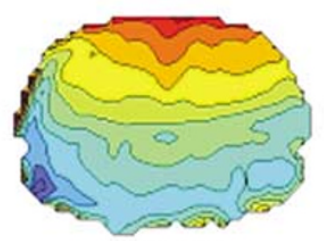

$\mathrm{t}=3.62$

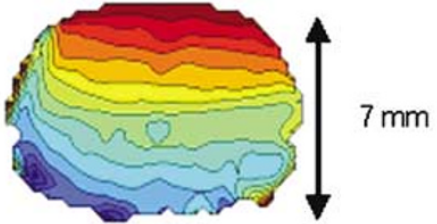

$\mathrm{t}=0.62$

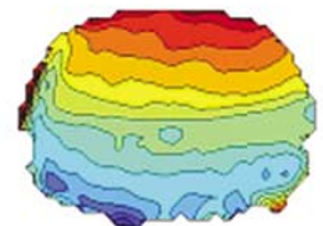

$\mathrm{t}=1.42$

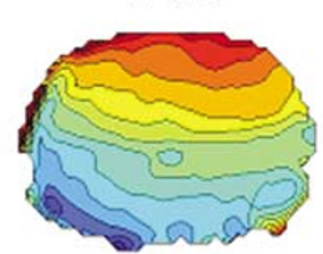

$\mathrm{t}=2.22$

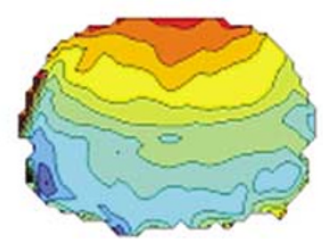

$\mathrm{t}=3.02$

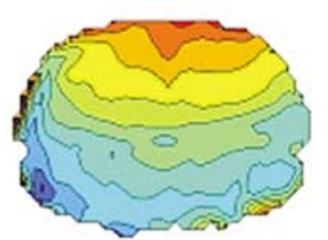

$\mathrm{t}=3.82$

Figure 3 Ocular surface height difference maps for subject DZ derived at time $t=0.02,0.22,0.42 \mathrm{~s} \ldots$. The height difference was derived by subtracting the map at $t=0$ immediately after blink from subsequent maps. Positive values (red) therefore indicate a relative thickening and negative values (blue) a relative thinning of the tear film. The colour bar represents the height scale $(\mu \mathrm{m})$.

of the map became lower compared with the baseline map at $t=0.62 \mathrm{~s}$ post-blink. Within $3.2 \mathrm{~s}$, at the superior edge of the map the relative surface height became lower by $1.4-2.8 \mu \mathrm{m}$ and at the same time became higher by $0.7-1.4 \mu \mathrm{m}$ at the inferior edge of the map.

For most of the subjects, the surface height initially increased at the superior edge of the map presumably owing to the upward movement of the tears after a blink following the path of the upper eye lid. The data showed greater variability in the 'inter-blink' phase, with no obvious change in the surface height being observed in a number of subjects.

\section{Changes in ocular surface wavefront aberrations}

All of the ocular surface wavefront aberration signals measured exhibited temporal fluctuations. We examined the group mean changes in the ocular surface wavefront aberrations. Group data of 'one-way' repeated measures ANOVA in ocular surface wavefront aberrations were calculated. Magnitudes of ocular surface horizontal coma (absolute values) and secondary astigmatism at $45^{\circ}$ significantly increased $(P<0.001$ and $P=0.007)$ in the inter-blink interval, and ocular surface secondary astigmatism at $0^{\circ}$ showed a significant decrease $(P=0.02)$ 


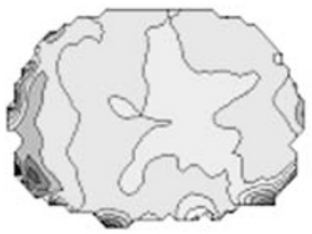

$t=0.02$

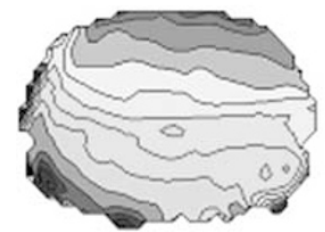

$\mathrm{t}=0.18$

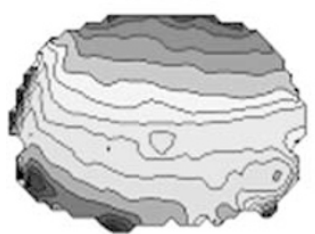

$t=0.34$

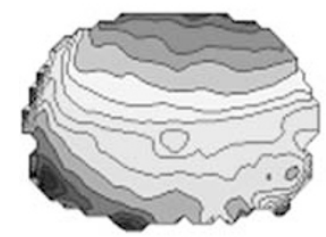

$\mathrm{t}=0.5$

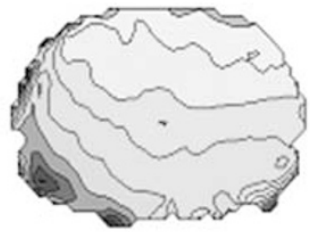

$\mathrm{t}=0.06$

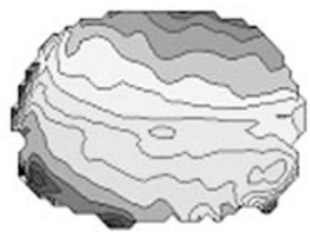

$t=0.22$

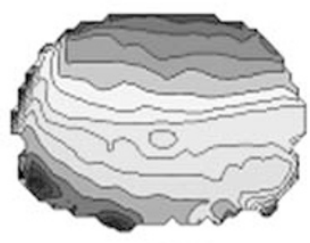

$t=0.38$

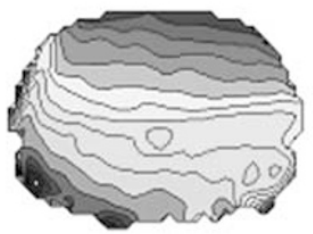

$t=0.54$

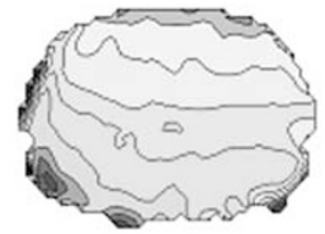

$\mathrm{t}=0.1$

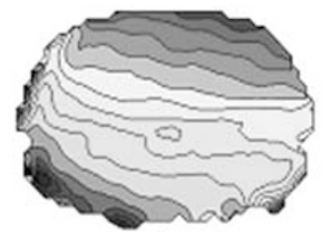

$t=0.26$

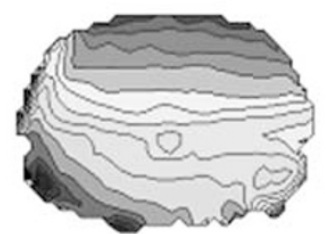

$\mathrm{t}=0.42$

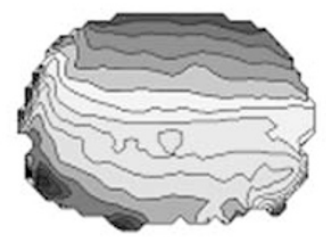

$\mathrm{t}=0.58$

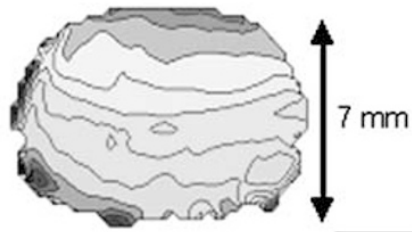

$\mathrm{t}=0.14$

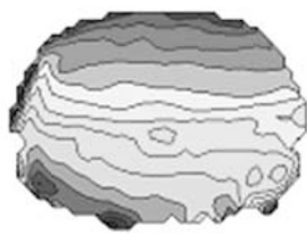

$\mathrm{t}=0.3$

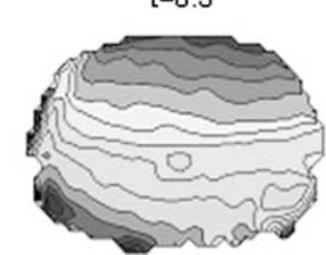

$\mathrm{t}=0.46$

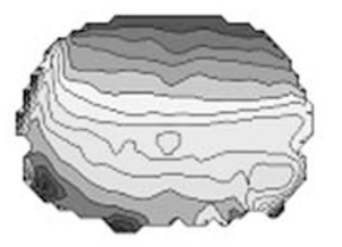

$\mathrm{t}=0.62$

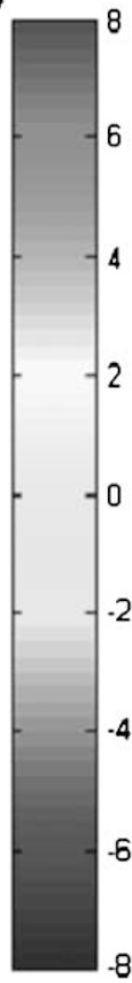

6

4

Figure 4 Ocular surface height difference maps for subject DZ derived at time $t=0.02,0.06,0.1 \mathrm{~s} \ldots$. The height difference was derived by subtracting the map at $t=0$ immediately after blink from subsequent maps. Positive values (red) therefore indicate a relative thickening and negative values (blue) a relative thinning of the tear film. The colour bar represents the height scale ( $\mu \mathrm{m})$.

in its magnitude. However, the other ocular surface Zernike components measured showed no significant changes (see Table 1).

The magnitude of fluctuations contained in the signals were also examined and the variance of the stable segments (ie ignoring the build-up phase) from all the inter-blink intervals for each ocular surface Zernike aberration were calculated. Figure 5 shows the group average standard deviation in each ocular surface Zernike coefficient. The prisms exhibited the largest variations among all the ocular surface Zernike wavefront aberrations measured. The microfluctuations in the astigmatisms, trefoil, and tetrafoil were found to be greater than those in defocus, comas, secondary astigmatism, and spherical aberration. The variations in ocular surface defocus were the lowest among the 14 ocular surface wavefront aberrations that were measured.

\section{Frequency domain representation}

To determine if there was a dominant frequency component contained within the measured signals, the power spectra of the ocular surface Zernike aberration signals, pulse, and instantaneous heart rates were estimated by using the FFT technique. With the 10 subjects' power spectra of ocular surface wavefront aberrations, no major peaks were found close to the pulse and instantaneous heart rate frequency locations. There were often low-frequency components $(<0.2 \mathrm{~Hz})$, which were likely to be caused by the changes in tear film thickness owing to the upward tear flow following blinking.

\section{Eye position}

Changes in the anterior-posterior eye position (apex distance) were examined and the power spectra were 
Table 1 Group data of 'one-way' repeated measures analysis of variance in ocular surface wavefront aberration components in the first $4.5 \mathrm{~s}$ after a blink

\begin{tabular}{|c|c|c|c|}
\hline \multirow[t]{2}{*}{ Zernike aberrations } & & \multicolumn{2}{|c|}{ ANOVA analysis } \\
\hline & & $F(d f=43)$ & P-value \\
\hline$\$ Z \_\{1\}^{\wedge}\{-1\} \$$ & Vertical prisom & 0.675 & 0.942 \\
\hline$\$ Z_{-}\{1\}^{\wedge}\{1\} \$$ & Horizontal prisom & 0.786 & 0.830 \\
\hline$\$ Z \_\{2\}^{\wedge}\{-2\} \$$ & Astigmatism $\left(45^{\circ}\right)$ & 1.033 & 0.420 \\
\hline$\$ Z_{-}\{2\}^{\wedge}\{0\} \$$ & Defocus & 0.745 & 0.881 \\
\hline$\$ Z \_\{2\}^{\wedge}\{2\} \$$ & Astigmatism $\left(0^{\circ}\right)$ & 0.554 & 0.990 \\
\hline$\$ Z \_\{3\}^{\wedge}\{-3\} \$$ & Trefoil $\left(90^{\circ}\right)$ & 1.186 & 0.206 \\
\hline$\$ Z_{-}\{3\}^{\wedge}\{-1\} \$$ & Vertical coma & 0.579 & 0.985 \\
\hline$\$ Z_{-}\{3\}^{\wedge}\{1\} \$$ & Horizontal coma & 2.119 & $<0.0001^{*}$ \\
\hline$\$ Z \_\{3\}^{\wedge}\{3\} \$$ & Trefoil $\left(0^{\circ}\right)$ & 0.942 & 0.579 \\
\hline$\$ Z_{-}\{4\}^{\wedge}\{-4\} \$$ & Tetrafoil $\left(22.5^{\circ}\right)$ & 0.906 & 0.642 \\
\hline$\$ Z_{-}\{4\}^{\wedge}\{-2\} \$$ & Sec astigmatism $\left(45^{\circ}\right)$ & 1.668 & $0.007^{*}$ \\
\hline$\$ Z \_\{4\}^{\wedge}\{0\} \$$ & Spherical aberration & 0.685 & 0.935 \\
\hline$\$ Z \_\{4\}^{\wedge}\{2\} \$$ & Sec astigmatism $\left(0^{\circ}\right)$ & 1.541 & $0.020^{*}$ \\
\hline$\$ Z \_\{4\}^{\wedge}\{4\} \$$ & Tetrafoil $\left(0^{\circ}\right)$ & 0.825 & 0.776 \\
\hline
\end{tabular}

Group data for nine subjects.

* indicates significant changes.

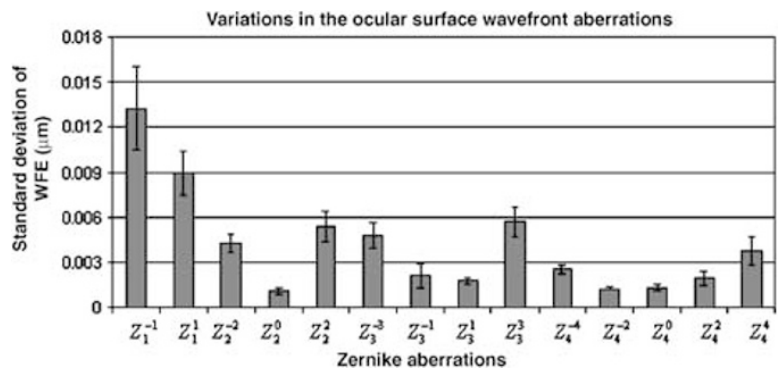

Figure 5 Group mean standard deviation of signals for each ocular surface Zernike wavefront aberration. Data are the mean of multiple signals for 10 subjects. Error bars are \pm 1 SD.

calculated. In Figure 6, we show the interpolated signal of the ocular surface apex distance (top) and the power spectra of the ocular surface apex distance plotted with the normalised spectra of the pulse and instantaneous heart rate signals (bottom) from subject DF. The average drift in the apex distance signal was approximately $0.2 \mathrm{~mm}$, with a maximum range of $0.52 \mathrm{~mm}$. Fluctuations of the ocular surface apex distance have two frequency components, which show agreement with the pulse frequency at $1.3 \mathrm{~Hz}$ and the instantaneous heart rate frequency at $0.5 \mathrm{~Hz}$. With the other nine subjects' power spectra of the apex distance, similar frequency spectra with two components at the pulse and instantaneous heart rate frequencies were typically observed. There are a number of other peaks in the power spectra that were not related to pulse or instantaneous heart rate, and their origin is unknown.

Temporal changes in the axis of asymmetric aberrations (such as astigmatism) were investigated to
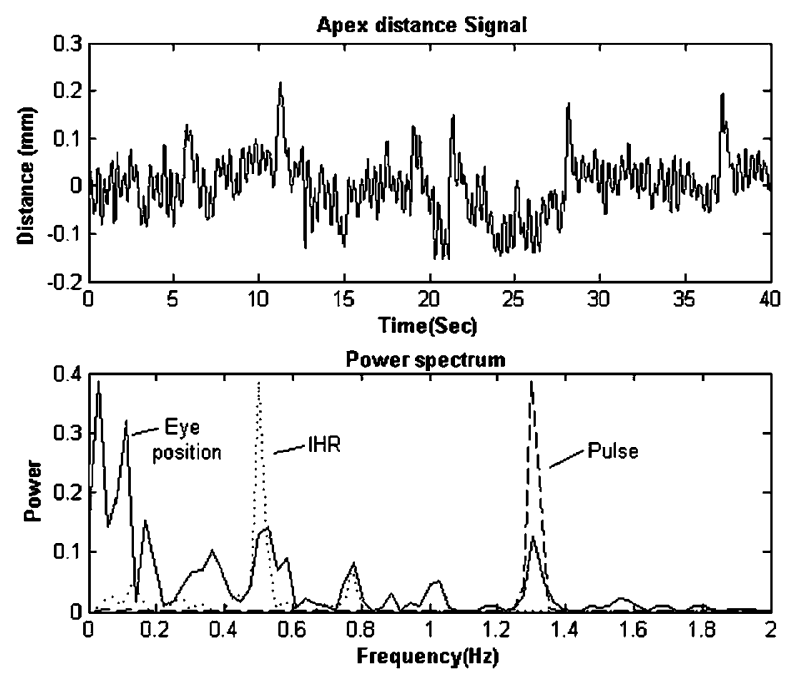

Figure 6 Raw signal of apex distance (top) and power spectrum of apex distance plotted with the scaled normalised power spectra of the subject's pulse and instantaneous heart rate (IHR) (bottom) for subject DF.

see if they were correlated with pulse rate owing to cyclotorsions in the ocular surface associated with pulsatile blood flow through the extra-ocular muscles. For all the subjects we examined, the power spectra of the astigmatism axis did not have a major frequency component that showed association with the pulse frequency.

\section{Discussion}

The results of the ocular surface height analysis over time showed that the tear film height increased at the superior cornea during the tear film 'build-up' phase. This was probably owing to the rapid upward movement of the tears after a blink, and we assume that this reflects a thickening of the tears in this region. It has been suggested that a thinner lipid layer in the superior cornea immediately after a blink causes a high surface tension, which results in upward drift of the tear film and then leads to a thicker tear film in the upper cornea. ${ }^{1,14}$ Owens and Phillips ${ }^{15}$ found that tear upward spreading velocity decreased to minimum one s post-blink. Our results also showed that the increase in ocular surface height in the superior region of the map in the 'build-up phase' was first very rapid (less than $1 \mathrm{~s}$ ) and then became relatively stable.

An important issue for achieving reliable estimates of corneal and total eye aberrations is the timing of measurements relative to the blink cycle. Buehren $e t a l^{16}$ found less ocular surface topography variability at $4 \mathrm{~s}$ post-blink, compared with 8 and $12 \mathrm{~s}$ post-blink. Nemeth et $a l^{4}$ and Montes-Mico $e t a l^{3}$ have suggested that 
videokeratoscopy measurement should be made at a fixed time of approximately $5 \mathrm{~s}$ after blink. Recently, Iskander et $a l^{10}$ estimated the tear film build-up time by using an RMS fit surface indicator based on Zernike polynomials. They estimated tear film build-up time to range between 1.5 and $7 \mathrm{~s}$. Based on the results from this study and previous studies, we suggest that clinical measurement of topography using videokeratoscopy should occur between 1 and $4 \mathrm{~s}$ after a blink to avoid the variability in results owing to the tear film build-up and break-up phases.

Our results on the inter-blink temporal changes in ocular surface height showed different trends with different subjects. We suspect that the potential factors causing this variation between subjects could be (1) continuing tear supply from under the upper lid, (2) tears leaving inter-palpebral space into the tear menisci and canaliculi (drainage system), (3) gravity effects, (4) tear evaporation, and (5) tear flow difference related to lid velocity or blink completeness. We found that changes in vertical coma showed good agreement with the changes in the tear ocular surface height despite the variations between subjects.

In our study, the videokeratoscopy data were used to estimate the ocular surface topography from which the surface height data were derived. Hence, the actual tear film thickness was not measured and only the relative topography of the tears was derived. The technique we have used cannot account for underlying changes in tear film thickness at the centre of the topography map and also assumes that the underlying corneal surface topography has not been changed by lid forces during blinking.

Both the low- and high-frequency components of microfluctuations in accommodation and individual Zernike aberrations have been found to be associated with pulse and instantaneous heart rate via the ocular pulse. ${ }^{17-19}$ In our investigation of the correlation between the microfluctuations in ocular surface wavefront aberrations and pulse and instantaneous heart rates, we found that they were not correlated. We believe that the microfluctuations in the ocular surface wavefront aberrations are primarily the results of the dynamics of the tear film and blinking ${ }^{20}$ rather than pulse and instantaneous heart rate. Given that the temporal critical fusion frequency of the eye is about $20 \mathrm{~Hz}$, the sampling frequency of $50 \mathrm{~Hz}$ used in this study should be fast enough to reveal dynamic changes in surface topography that might affect vision.

Variations in fixation owing to the natural microfluctuations in eye position have been reported to have an effect on ocular surface topography measurement by videokeratoscopy. ${ }^{21}$ The fluctuations in the ocular surface apex distances in our example
(Figure 6) showed magnitudes similar to those previously reported ${ }^{22,18}$ and contained two frequency components showing agreement with the pulse and instantaneous heart rate frequencies. However, this anterior-posterior ocular movement should not affect the data collected from the Medmont videokeratoscope because the topography calculation algorithm is based on the measured apex distance. If anterior-posterior movements of the eye were not corrected, then Zernike aberrations such as defocus and spherical aberration terms derived from the topography would have shown pulse-related frequencies. Hence, the changes in ocular surface Zernike wavefront aberrations we found are most likely owing to the dynamics in the tear film and not ocular micro-movements ( $x, y$, or $z$ direction). We also examined the changes in the pupil central displacement in both $x$ and $y$ directions and found that the impact of pulse and respiration was much smaller in the $x$ or $y$ direction (left-right and up-down) than in the $z$ direction (apex distance). Furthermore, we did not find any cyclic changes in the axis of asymmetric astigmatism aberrations that could correlate with pulse rate and hence it is not likely that there were significant cyclo-torsions in the ocular surface associated with pulse.

In conclusion, we found that the ocular surface height at the upper edge of the topography map increased by approximately $2 \mu \mathrm{m}$ within $0.5 \mathrm{~s}$ post-blink. There are few ocular surface wavefront aberration components that showed significant changes in the inter-blink interval. Fluctuations in the ocular anterior-posterior movement could be partially correlated with pulse and instantaneous heart rate frequencies. We suggest that the clinical measurement of corneal topography using videokeratoscopy should occur within the period 1-4 s after a blink to improve reliability.

\section{References}

1 Benedetto DA, Clinch TE, Laibson PR. In vivo observation of tear dynamics using fluorophotometry. Arch Ophthal 1984; 102: 410-412.

2 Nemeth J, Erdelyi B, Csakany B, Gaspar P, Soumelidis A, Kahlesz F, Lang Z. High-speed videotopographic measurement of tear film build-up time. Invest Ophthal Vis Sci 2002; 43(6): 1783-1790.

3 Montes-Mico R, Alio JA, Munoz G, Charman WN. Temporal changes in optical quality of air-tear film interface at anterior cornea after blink. Invest Ophthal Vis Sci 2004a; 45(6): 1752-1757.

4 Nemeth J, Erdelyi B, Csakany B. Corneal topography changes after $15 \mathrm{~s}$ pause in blinking. J Cataract Refract Surg 2000; 27: 589-592.

5 Montes-Mico R, Alio JA, Munoz G, Perez-Santonja J, Charman WN. Postblink changes in total and corneal ocular aberrations. Ophthalmology 2004b; 111(4): 758-767. 
6 Goto T, Zheng X, Klyce SD, Kataoka H, Uno T, Karon $\mathrm{M}$ et al A new method for tear film stability analysis using videokeratography. Am J Ophthal 2003; 135(5): 607-612.

7 Goto T, Zheng X. Tear flim stability analysis system: introducing a new application for videokeratography. Cornea 2004; 23(8): s65-s70.

8 Kojima T, Ishida R, Dogru M, Goto T, Takano Y, Matsumoto $\mathrm{Y}$ et al A new noninvasive tear stability analysis system for the assessment of dry eyes. Invest Ophthalmol Vis Sci 2004; 45(5): 1369-1374.

9 Montes-Mico R, Alio JA, Charman WN. Dynamic changes in the tear film in dry eyes. Invest Ophthal Vis Sci 2005; 46(5): $1615-1619$

10 Iskander DR, Collins MJ, Davis B. Evaluating tear film stability in the human eye with high speed videokeratoscopy. IEEE Trans Biomed Eng 2005; 52(11): 1939-1949.

11 Iskander DR, Collins MJ. Applications of high speed videokeratoscopy. Clin Exp Optom 2005; 88(4): 223-231.

12 Friesen GM, Jannett TC, Jadallah MA, Yates SL, Quint SR, Nagle HT. A comparison of the noise sensitivity of nine QRS detection algorithms. IEEE Trans Biomed Eng 1990; 37: 85-98.

13 Iskander DR, Collins MJ, Morelande MR, Zhu M. Analyzing the dynamic wavefront aberrations in the human eye. IEEE Trans Biomed Eng 2004; 51(11): 1969-1980.
14 King-Smith PE, Fink BA, Hill RM, Koelling KW, Tiffany JM. The thickness of the tear film. Curr Eye Res 2004; 29(4-5): 357-368.

15 Owens H, Phillips J. Spreading of the tears after a blink. Cornea 2001; 20(5): 484-487.

16 Buehren T, Collins MJ, Iskander DR, Davis B, Lingelbach B. The stability of corneal topography in the post-blink interval. Cornea 2001; 20(8): 826-833.

17 Winn B, Pugh JR, Gilmartin B, Owens B. Arterial pulse modulates steady-state ocular accommodation. Curr Eye Res 1990; 9(10): 971-975.

18 Collins MJ, Davis B, Wood J. Microfluctuations of steadystate accommodation and the cardiopulmonary system. Vis Res 1995; 35(17): 2491-2502.

19 Zhu M, Collins MJ, Iskander DR. Microfluctuations of wavefront aberrations of the eye. Ophthal Physiol Opt 2004; 24(6): 562-571.

20 Tsubota K, Nakamori K. Effect of ocular surface area and blink rate on tear dynamics. Arch Ophthalmol 1995; 113(2): 155-158.

21 Buehren T, Lee BJ, Collins MJ, Iskander DR. Ocular microfluctuations and videokeratoscopy. Cornea 2002; 21(4): 346-351.

22 Collins MJ, Smyth W, Seawright J, Kelly S. The synkinesis between antero-posterior eye position and lid fissure width. Clin Exp Opt 1992; 75(2): 38-41. 